Acta Botanica Mexicana (2004), 66: 1-22

\title{
ADICIONES AL CONOCIMIENTO DE LA DIVERSIDAD DE LOS HONGOS CONIDIALES DEL BOSQUE MESÓFILO DE MONTAÑA DEL ESTADO DE VERACRUZ
}

\author{
Gabriela Heredia Abarca, Manuela Reyes Estebanez y Rosa Ma. Arias Mota \\ Instituto de Ecología, A.C. \\ Carretera Antigua a Coatepec 351 \\ Congregación El Haya, 91070 Xalapa, Veracruz, México \\ heredia@ecologia.edu.mx \\ Julio Mena Portales y Ángel Mercado Sierra \\ Instituto de Ecología y Sistemática \\ La Habana, Cuba
}

\begin{abstract}
RESUMEN
En la presente contribución se describen e ilustran 29 especies de hongos conidiales en su fase anamorfa y una condición holomorfa. Todos los hongos estudiados son saprobios de restos vegetales colectados en diversas localidades con vestigios de bosque mesófilo de montaña del estado de Veracruz. Hasta la fecha el material que se presenta era desconocido para la micobiota de México. En su mayoría son especies raras, cuyo registro a nivel mundial ha sido escaso. Se anexan datos sobre su distribución y sustratos en que proliferan. La identificación se basó en la morfología de los hongos tal como se desarrollan sobre el sustrato en que fueron colectados. El material de referencia está depositado en la colección de hongos conidiales del Instituto de Ecología, A.C. (XAL) en Xalapa, Veracruz, México.
\end{abstract}

Palabras clave: bosque mesófilo de montaña, fase anamorfa, hongos conidiales, hongos saprobios, México, Veracruz.

\begin{abstract}
An account of 29 anamorphs and one holomorph species are presented, all of them were collected in several localities of the State of Veracruz, where small areas of cloud forest still remain. All the species are recorded for the first time to Mexico, many of them are scarcely known in the worldwide. Pictures and data about their distribution and substrates are included. Identification was based on the morphology of the fungi growing directly on naturally occurring plant debris. Specimens are deposited at the conidial fungi collection of the Instituto de Ecología, A.C. (XAL) in Xalapa, Veracruz, México.
\end{abstract}

Key words: anamorph phase, cloud forest, conidial fungi, Mexico, saprobic fungi, Veracruz. 


\section{INTRODUCCIÓN}

Por sus características estructurales y por las condiciones climatológicas en que se desarrollan, los bosques mesófilos de montaña (BMM) son ecosistemas en donde prolifera una vasta diversidad fúngica. Para México, varias contribuciones han expuesto la riqueza de especies macroscópicas que prosperan en este hábitat (Chacón y Medel, 1993), no así para los hongos microscópicos, de los cuales aún existe un precario conocimiento. Entre éstos, sobresalen por su abundancia y diversidad las especies conidiales. En los BMM la acumulación de restos vegetales y la alta humedad relativa imperante durante todo el año favorecen el desarrollo de especies saprobias, de tal forma que los hongos conidiales constituyen una comunidad microscópica bien representada, cuya composición y estructura todavía están lejos de ser comprendidas.

A los hongos conidiales también se les denomina hongos mitospóricos, hongos imperfectos y/o hongos anamorfos, como consecuencia del desconocimiento de su fase sexual (Hawksworth et al., 1995); son muy pocas las especies conidiales a las que se les conoce su fase teleomorfa o sexual, la conexión entre anamorfos y teleomorfos es uno de los retos a los que se enfrentan los micólogos y cuyas implicaciones tienen relevancia a nivel filogenético. Se emplea el término holomorfo a aquellas especies en las que se ha logrado conocer tanto la fase asexual como sexual del hongo (Hennebert y Weresub, 1979).

La importancia de estos organismos no se limita al papel que juegan en el equilibrio ecológico como degradadores de restos orgánicos y reguladores de poblaciones fitopatógenas, sino que además tienen el potencial de producir enzimas y metabolitos secundarios importantes en las industrias farmacológica y alimenticia entre otras.

Ante las constantes prácticas de deforestación resulta imprescindible la exploración micológica de las zonas en donde aún existe la flora característica de los BMM. Los resultados que a continuación se presentan surgen de las colectas que han efectuado los autores dentro de varios proyectos encaminados a la realización de un inventario de los hongos conidiales saprobios del estado de Veracruz.

El objetivo de esta contribución es incrementar el conocimiento de la diversidad de los hongos conidiales saprobios para México en general y en particular para el bosque mesófilo de montaña.

\section{MATERIALES Y MÉTODOS}

El material colectado consistente en ramas, hojas, troncos e inflorescencias caídas, se colocó en cámaras húmedas, las cuales se revisaron periódicamente bajo el microscopio. De todas las especies se realizaron preparaciones permanentes en alcohol polivinil. La identificación se efectuó utilizando la bibliografía especializada 
para cada caso. El material de referencia está depositado en la colección de hongos conidiales del Instituto de Ecología, A.C.

\section{DESCRIPCIONES DE LAS ESPECIES}

Acremonium anamorfo de Wallrothiella subiculosa Höhn., 1912, Sitzungsber. Akad. Wiss. Wien, 121: 381.

三 Torula protea Sacc., 1881, Michelia, 2: 292.

$\equiv$ Gliomastix protea (Sacc.) Verona et Castella, 1942, Annal. Fac. Agr. Univ.

Pisa, n. s. 5: 383.

Conidióforos erectos, rectos o flexuosos, simples, de color café claro, lisos, con un collarín en la parte apical, 37-63 x 2.5-4 $\mu \mathrm{m}$. Células conidiógenas monofialídicas. Conidios elipsoidales, formando cabezas mucilaginosas esféricas en el ápice del conidióforo, pueden presentar la base redondeada o truncada, lisos, aseptados, de color café pálido, 5.5-7 x 3-4 $\mu \mathrm{m}$. Fig. 17.

Materiales examinados: Sobre tronco herbáceo, mpio. Cosautlán, Cosautlán, col. J. Mena-Portales, 6-VII-95, CB484.

Distribución conocida: Muy probablemente se trate de un hongo cosmopolita; crece sobre una gran variedad de restos vegetales, también se ha aislado del suelo. Dickinson (1968), Ellis (1971) y Mercado-Sierra et al. (1997a) incluyen una amplia lista de países de donde ha sido registrada la especie.

Alysidiopsis pipsissewae B. Sutton, 1973, Mycol. Pap. 132: 5-8.

Conidióforos erectos, rectos o ligeramente flexuosos, lisos, septados, de color café oscuro, más claros en la parte apical, 110-419 x 7-16 $\mu \mathrm{m}$. Células conidiógenas integradas, monoblásticas y poliblásticas, terminales. Conidios esféricos, ovales, elipsoidales, septos 0-1, lisos, de color café pálido, 4.5-9 x 3-5 $\mu \mathrm{m}$. Figs. 1a, b.

Materiales examinados: Sobre Bromelia, mpio. Ixhuacán de Los Reyes, Ixhuacán de Los Reyes, col. G. Olvera, 5-III-95, CB546-1. En tallos caídos, mpio. Xicochimalco, Cascadas de Texolo, col. A. Mercado-Sierra, 11-II-96, CB644-1. En tallos caídos, mpio. Xicochimalco, Cascadas de Texolo, col. G. Olvera, 2-XII-95, CB649.

Distribución conocida: Canadá, sobre rama muerta de Chimaphila umbellata (Sutton, 1973). 
Beltrania malaiensis G.E.M. Wakefield, 1931, Kew Bull. 4: 204.

Setas simples, erectas, lisas, de color café oscuro, hasta $205 \mu \mathrm{m}$ de largo, célula basal de 13-15 $\mu \mathrm{m}$ de diámetro. Conidióforos simples, erectos, rectos 0 ligeramente flexuosos, lisos, de color café pálido, 105-184 x 4-4.5 $\mu \mathrm{m}$. Células separadoras elipsoidales, hialinas, 10-12 x 7-9 $\mu \mathrm{m}$. Conidios rómbicos, lisos, con una banda transversal hialina, café oliváceos, 27-36 x 17-19 $\mu \mathrm{m}$, apéndice 9-12 $\mu \mathrm{m}$. Fig. 4.

Materiales examinados: Sobre hojarasca de Quercus sp., mpio. Xalapa, Rancho Guadalupe, cols. G. Heredia, R. M. Arias y M. Reyes, 29-XI-01, CB752.

Distribución conocida: Malasia, sobre semillas de Dichopsis guta (Hughes, 1951), y Brasil, sobre hojarasca de Miconia cabussus (Gusmao, 2001).

Botryosporium longibrachiatum (Oudem.) Maire, 1903, Ann. Mycol., Berl. 1: 341.

$\equiv$ Botrytis (Phymatotrichum) longibrachiatum Oudem.,1890, Micromycetes nouveaux, p. 7

$\equiv$ Botryosporium pulchellum Maire, 1900, Bull. Soc. Sci. Nancy 100: 161-163.

$\equiv$ Cephalosporium dendroides M.B. Ellis et Kellerman in Kellerman, 1903. J. Mycol. 9: 5 .

$\equiv$ Redaisiella elegans Bainier, 1910, Bull. Soc. Mycol. Fr. 26: 382.

Conidióforos rectos, lisos, hialinos, ramificados lateralmente, ensanchándose hacia la parte apical en forma de rombo. Células conidiógenas formadas por 2-5 lóbulos, hialinas, denticuladas en la parte superior. Conidios ovoides, elipsoidales, ligeramente rugosos, hialinos, 6-7 × 2-2.4 $\mu \mathrm{m}$. Fig. 2 .

Materiales examinados: Sobre hojarasca de Quercus sp., mpio de Xalapa. Rancho Guadalupe, col. G. Bills, 6-VI-00, CB753.

Distribución conocida: Ghana, Sierra Leona, Pakistán, Malasia (Hughes, 1953), Canadá (Barron, 1977), Inglaterra (Thian-Yu y Kendrick, 1990) y Cuba (Minter et al., 2001). Se ha colectado en restos vegetales y en suelo.

Catenularia kalakadensis Subram. et D.J. Bhat, 1987, Kavaka 15(1, 2): 49.

Conidióforos erectos, rectos o flexuosos, lisos, septados, con proliferaciones percurrentes, de color café claro, 105-162 x 3-5 $\mu \mathrm{m}$. Células conidiógenas integradas, monofialídicas, terminales, con un collar apical, de color café claro, 
lisas, $19-30 \times 3-4.5 \mu \mathrm{m}$. Conidios cuneiformes, angulados distalmente, lisos, con la base truncada, de color café pálido, 5.5-7.5 x 4-5 $\mu \mathrm{m}$. Figs. 24 a, b.

Materiales examinados: Sobre tronco muerto, mpio. Xalapa, Rancho Guadalupe, col. J. Mena-Portales, 10-VI-95, CB365-2.

Distribución conocida: La India, sobre ramas muertas no identificadas (Subramanian y Bhat, 1987).

Cladosporium vignae Gardner, 1925, Phytopathology 15: 457.

Conidióforos erectos, rectos, lisos, hialinos, 39-91 x 3-5 $\mu \mathrm{m}$. Células conidiógenas holoblásticas, poliblásticas, integradas, terminales o intercaladas, con cicatrices prominentes. Conidios cilíndricos, claviformes, en cadenas simples 0 ramificadas, septos $0-2$, lisos, de color café pálido, con 2 ó 3 cicatrices, 20-33 x 5-6 $\mu \mathrm{m}$; además presenta conidios elipsoidales, en forma de limón, lisos, hialinos, $6-16 \times 5-6 \mu \mathrm{m}$. Fig. 3 .

Materiales examinados: Sobre inflorescencia de Erythrina sp., mpio. Xalapa, Parque Ecológico "El Haya", cols. G. Heredia, R. M. Arias y M. Reyes, 12-IX-01, CB756-1, CB756-2, CB756-3.

Distribución conocida: C. vignae ha sido descrita para varias localidades de Estados Unidos, el material tipo se extrajo de vainas de Vigna unguiculata (MorganJones y McKemy, 1992).

Cordana crassa Tóth, 1975, Ann. Hist.-Nat. Mus. Nat. Hung. 67: 32.

$\equiv$ Cordana boothii M.B. Ellis, 1976, More Dematiaceous Hyphomycetes, p. 162.

Conidióforos erectos, rectos o flexuosos, lisos, septados, de color café oscuro, pálidos hacia el ápice, hasta $154 \mu \mathrm{m}$ de long. x 5-9 $\mu \mathrm{m}$ en la parte basal, parte apical globosa, 11.5-12 $\mu \mathrm{m}$. Células conidiógenas monoblásticas, integradas. Conidios obpiriformes, con un septo, lisos, la célula basal café oscura y la apical de color café claro, 17.5-20 x 11-12 $\mu \mathrm{m}$. Fig. 11.

Materiales examinados: Sobre madera, mpio. Huatusco, Las Cañadas, cols. G. Heredia y R. Castañeda, 26-VII-99, CB757. 1976).

Distribución conocida: Inglaterra y Hungría, sobre restos de madera (Ellis, 
Cordana musae (Zimm.) Höhn., 1923, Zentbl. Bakteriol. Parasitenk. Abt. 2, 60: 7. EScolecotrichum musae Zimm., 1902, Zentbl. Bakteriol. Parasitenk. Abt. 2, 8: 220.

Conidióforos erectos, rectos o flexuosos, lisos, de color café pálido, 153-158 x 6-7.5 $\mu \mathrm{m}$, ensanchados en la base hasta de $11 \mu \mathrm{m}$ de diámetro. Conidios piriformes, con un septo, lisos, hialinos a cafés pálidos, 14-18 x 7-18 $\mu \mathrm{m}$. Fig. 7.

Materiales examinados: Sobre hojarasca, mpio. Xalapa, Rancho Guadalupe, cols. G. Heredia, R. M. Arias y M. Reyes, 29-XI-01, CB758.

Distribución conocida: Polonia (Czeczuga y Orlowska, 1997), la antigua Rusia (Melnik, 2000), Taiwan (Matsushima, 1980), Cuba, Jamaica, República Dominicana y Puerto Rico (Minter et al., 2001). Prolifera sobre restos foliares, con frecuencia está asociada a hojas del género Musa.

Chaetopsina splendida B. Sutton et Hodges, 1976, Nova Hedwigia 27(172): 346.

Setas erectas, ligeramente curvas, verruculosas, septadas, compuestas de una rama principal con terminación en punta, 191-218.5 x 4.5-5.5 $\mu \mathrm{m}$, y 4-5 ramas divergentes de 50-58 $\mu \mathrm{m}$ de largo $\times 4-5 \mu \mathrm{m}$ de ancho en la base, las cuales surgen de la parte media de la seta principal. Conidióforos inconspicuos, hialinos, compactados en una empalizada, situados por debajo de las ramas y rodeando la seta principal. Células conidiógenas enteroblásticas, fialídicas, discretas, ampuliformes, 4-7 x 2.5-4.5 $\mu \mathrm{m}$. Conidios cilíndricos, con los extremos redondeados, hialinos, aseptados, 9-12 x 1-1.5 $\mu \mathrm{m}$. Fig. 6 .

Materiales examinados: Sobre hojarasca, mpio. Coatepec, Rancho Viejo, cols. G. Heredia y J. Mena-Portales, 18-V-02, CB759-1, CB759-2, CB759-3.

Distribución conocida: Brasil, sobre hojas de Eucalyptus sp. (Sutton y Hodges, 1976) y Australia, sobre hojas no identificadas (Kirk y Sutton, 1985).

Dictyochaeta anamorfo de Chaetosphaeria dingleyae S. Hughes, Kendr. et Shoemaker, 1968, New Zeal. J. Bot. 6: 323-375.

Setas erectas, rectas, septadas, lisas, de color café oscuro, pálidas hacia el ápice, de 126-218.5 $\mu \mathrm{m} \times$ 4-6 $\mu \mathrm{m}$, parte apical redondeada, $3.5 \mu \mathrm{m}$ de ancho. Conidióforos macronematosos, rectos o ligeramente flexuosos, en grupos rodeando la seta, lisos, de color café pálido, 51.5-69 x 3-3.5 $\mu \mathrm{m}$. Células conidiógenas fialídicas, terminales e intercaladas, collarines prominentes. Conidios fusiformes, 
cilíndricos, curvos, extremos redondeados, con un septo, lisos, hialinos, 10-17 x 1.5$2 \mu \mathrm{m}$, con una sétula a cada lado, 6-12 $\mu \mathrm{m}$ de largo. Figs. $14 \mathrm{a}$, b.

Materiales examinados: Sobre hojas caídas de Quercus xalapensis, mpio. Huatusco, Las Cañadas, col. G. Heredia y R. Castañeda, 26-VII-99, CB760.

Distribución conocida: Nueva Zelanda, sobre madera (Hughes y Kendrick 1968).

Dischloridium laeense (Matsush.) B. Sutton, 1976, Kavaka 4: 47.

$\equiv$ Chloridium laeense Matsush., 1971, Bull. Nat. Sci. Mus. Tokyo 14(3): 462.

Conidióforos solitarios o en grupos, surgen de un estroma inmerso, rectos, de color café oscuro, pálidos en la parte apical, lisos, septados, con proliferaciones sucesivas, 188-353 x 5-9 $\mu \mathrm{m}$. Células conidiógenas fialídicas, hialinas, terminales, hasta de $70 \mu \mathrm{m}$ de largo, collarines inconspicuos. Conidios cilíndricos, elipsoidales, aseptados, gutulados, ligeramente truncados en la base, hialinos, 19.5-25 x 6-8 $\mu \mathrm{m}$ de ancho. Fig. 13.

Materiales examinados: Sobre hojarasca, mpio. Coatepec, Rancho Viejo, cols. G. Heredia y J. Mena-Portales, 18-V-02, CB761-1, CB761-2.

Distribución conocida: Especie pantropical, crece sobre hojas muertas de una amplia variedad de plantas; ha sido colectada en Papua-Nueva Guinea, (Matsushima, 1971), Sri Lanka (Sutton, 1976), Cuba (Holubová-Jechová, 1982a; Mena-Portales y Mercado-Sierra, 1987; Mercado-Sierra et al., 1997a), Australia, Etiopía, India, Malasia (Bath y Sutton, 1985), Inglaterra (Kirk, 1986), Taiwan (Matsushima, 1987) y Perú (Matsushima, 1993).

Edmundmasonia pulchra Subram., 1958, J. Ind. Bot. Soc. 37(3): 402-407.

Conidióforos erectos, rectos, flexuosos, septados, lisos, de color café oscuro, 197-269 x 7-10 $\mu \mathrm{m}$. Células conidiógenas terminales e intercaladas, monotréticas, de color café oscuro, ovoides, piriformes. Conidios claviformes, con el ápice redondeado, generalmente con 3 septos, lisos, de color café oscuro, 16-22 x 9$16 \mu \mathrm{m}$ en la parte más ancha. Fig. 8.

Materiales examinados: Sobre hojarasca, mpio. Xalapa, Rancho Guadalupe, col. J. Mena-Portales, 10-VI-95, CB351-2. 
Distribución conocida: La India, sobre hojas caídas (Subramanian, 1958), Japón, sobre hojas de Phyllostachys edulis y Bambusa multiplex, Australia y Ecuador, sobre hojas y peciolos de palma respectivamente (Matsushima, 1975, 1989 y 1993).

Ellisembia adscendens (Berk.) Subram., 1992, Proc. Indian Natn. Sci. Acad. B 58(4): 183.

$\equiv$ Sporidesmium adscendens Berk., 1840, Ann. Nat. Hist. 4: 291.

Conidióforos solitarios o en grupos, erectos, rectos o ligeramente flexuosos, lisos, septados, de color café rojizo, 21-66 x 4.5-7 $\mu \mathrm{m}$. Conidios obclaviformes, flexuosos, alargados, ápice redondeado y base truncada, presentan 15-33 distoseptos, lisos, café rojizos, hasta $230 \mu \mathrm{m}$ de largo x 11-25 $\mu \mathrm{m}$ de ancho. Fig. $27 a, b$.

Materiales examinados: Sobre tronco caído, mpio. Xalapa, Rancho Guadalupe, col. J. Mena-Portales, 10-VI-95, CB373-2. Sobre peciolo de palma, mpio. Comapa, Comapa, col. J. Mena-Portales, 16-VI-95, CB435-3. Sobre ramas muertas, mpio. Coatepec, La Pitaya, col. G. Rosas, 18-XI-95, CB 514-2. Sobre tronco muerto, mpio. Ixhuacán de Los Reyes, Ixhuacán de Los Reyes, col. A. Mercado-Sierra, 4-III-96, CB588-2.

Distribución conocida: Especie cosmopolita, preferentemente lignícola. Ampliamente distribuida en zonas tropicales. Existen numerosos registros de colectas. Detalles de su distribución en el Caribe pueden consultarse en Minter et al., (2001), autores adicionales que incluyen información para otras regiones son: Matsushima, 1975, 1980; Morris, 1978; Rao y Varghese, 1981; Rao y de Hoog, 1986; Cabello et al., 1993; Sutton, 1993 y McKenzie, 1995.

Eversia parvula Hol.-Jech., 1987, Céská Mykologie 41(1): 31-33.

Conidióforos simples o ramificados, rectos o ligeramente flexuosos, septados, de color café pálido, 9-18 x 3-4 $\mu \mathrm{m}$. Células conidiógenas holoblásticas, integradas, terminales, cilíndricas con 1 a 4 proliferaciones percurrentes, formando un anillo café oscuro en la parte apical del conidióforo. Conidios acrógenos, oblongos, aplanados, muriformes, de color café pálido a café oscuro, 18.5-26 x 7-9 $\mu \mathrm{m}$. Fig. 9.

Materiales examinados: Sobre madera, mpio. Coatepec, Rancho Viejo, cols. G. Heredia y J. Mena-Portales, 18-V-02, CB762-1, CB762-2, CB762-3.

Distribución conocida: Cuba, sobre ramas muertas (Holubová-Jechová, 1987; Holubová-Jechová y Mercado-Sierra, 1989) y antigua Unión Soviética (Melnik, 2000). 
Exserticlava vasiformis (Matsush.) S. Hughes., 1975, New Zeal. J. Bot. 16: 332-333. Cordana vasiformis Matsush., 1975, Icones Microfungorum a Matsushima Lectorum, p. 40.

Conidióforos erectos, rectos o ligeramente flexuosos, lisos, septados, de color café oscuro, pálidos hacia el ápice, 95-228 x 6-7.5 $\mu \mathrm{m}$, parte superior globosa, de 12-15 $\mu \mathrm{m}$ de ancho, a veces se forma una extensión del conidióforo, subulada, de pared lisa, pálida y con la parte apical redondeada. Células conidiógenas en forma de copa, lisas, cafés en la parte basal, más pálidas hacia el ápice, 18-20 $\mu \mathrm{m}$. Conidios elipsoidales, de color café pálido, con 3 distoseptos, lisos, 24-25 x 13$15 \mu \mathrm{m}$. Figs. 29 a, b.

Materiales examinados: Sobre ramas, mpio. Coatepec, Rancho Viejo, col. G. Heredia y J. Mena-Portales, 18-V-02, CB763-1, CB763-2.

Distribución conocida: Japón (Matsushima, 1971), Nueva Zelanda (Hughes, 1978), Taiwan (Matsushima, 1980), Estados Unidos (Crane y Schocknecht, 1982), Cuba (Castañeda, 1985), Perú (Matsushima, 1993), Malasia (Kuthubutheen y Nawawi, 1994), Costa Rica (Mercado-Sierra et al., 1997b), Puerto Rico (Melnik, 1998) y en la antigua Unión Soviética (Melnik, 2000). Prospera sobre sustratos herbáceos y lignícolas.

Gyrothrix podosperma (Corda) Rabenhorst var. falcatispora Mercado-Sierra et J. Mena-Portales, 1986, Acta Bot. Hung. 32(1-4): 195-196.

Setas erectas, ligeramente rugosas, septadas, ramificadas dicotómicamente en la parte superior, de color café oscuro en la base aclarándose hacia la parte superior, hasta $188 \mu \mathrm{m}$ de alto $\times 5-5.5 \mu \mathrm{m}$ de ancho en la base. Células conidiógenas obclaviformes, lageniformes, lisas, hialinas, 9-11 x 2.5-3.5 $\mu \mathrm{m}$. Conidios falciformes ligeramente curvos, lisos, sin septos, hialinos, 13-15 x 1-1.5 $\mu \mathrm{m}$. Fig. 12.

Materiales examinados: Sobre hojarasca, mpio. Xalapa, Rancho Guadalupe, cols. G. Heredia, R. M. Arias y M. Reyes, 29-XI-01, CB764-1, CB764-2.

Distribución conocida: Cuba, sobre hojarasca (Mercado-Sierra y MenaPortales, 1986).

Hyphodiscosia jaipurensis Lodha et K.R.C. Reddy, 1974, Trans. Br. Mycol. Soc. 62 : 418-421.

Conidióforos erectos, simples, septados, de color café pálido, hialinos en el ápice, lisos, 67-81 x 5-6.5 $\mu \mathrm{m}$ de ancho. Células conidiógenas integradas, 
terminales, poliblásticas, clavadas, denticuladas. Conidios cilíndricos, con el ápice redondeado, lisos, hialinos, septados, 20-22.5 x 3.5-4.5 $\mu \mathrm{m}$, con 2 sétulas cerca de la parte media del septo hasta de $12 \mu \mathrm{m}$. Fig. 10.

Materiales examinados: Sobre ramas caídas, mpio. de Xalapa, Jardín Botánico Francisco Javier Clavijero, cols. G. Heredia, R. M. Arias y M. Reyes, 30XI-01, CB765-1, CB765-2.

Distribución conocida: Japón (Matsushima, 1975), India (Ellis, 1976), Guam (Matsushima, 1981) y Cuba (Castañeda y Arnold, 1985). Crece sobre semillas, corteza, hojas y ramas muertas.

Idriella rara R. F. Castañeda, 1985, Deuteromycotina de Cuba. Hyphomycetes II: 6.

Conidióforos erectos, rectos, septados, ramificados en la parte superior, lisos, de color café oscuro en la base, hialinos en la parte superior, 65-100 x 4-6 $\mu \mathrm{m}$ de ancho. Células conidiógenas poliblásticas, ampuliformes, integradas, denticuladas, 9-15 x 3-4 $\mu \mathrm{m}$. Conidios falciformes, lisos, septos 0 ó 1, hialinos $26-33 \times 1.5-2 \mu \mathrm{m}$. De las células conidiógenas se producen cristales cilíndricos, rectos, hialinos, de 22-27 x 0.5-1 $\mu \mathrm{m}$. Fig. 19.

Materiales examinados: Sobre hojas muertas, mpio. de Xalapa, Rancho Guadalupe, cols. G. Heredia, R. M. Arias y M. Reyes. 29-XI-01, CB766.

Distribución conocida: Cuba (Castañeda, 1985; Mercado-Sierra et al., 1998; Minter et al., 2001). Sobre hojas muertas.

Masoniomyces claviformis Crane et Dumont, 1975, Can. J. Bot. 53: 847-849.

Conidióforos simples o ramificados, rectos o flexuosos, pared delgada, septados, de color café oliváceo, lisos, claviformes en la parte superior, hasta de $900 \mu \mathrm{m}$ de largo y 7-11 $\mu \mathrm{m}$ de ancho en la base, 14-16 $\mu \mathrm{m}$ en la parte superior. Células conidiógenas discretas, acropleurógenas, poliblásticas, cubriendo la parte claviforme del conidióforo. Conidios fusiformes, elípticos, obtusos en el ápice y con la base truncada, aseptados, lisos, subhialinos, 5-7.5 x 1.5-2.5 $\mu \mathrm{m}$. Fig. 20.

Materiales examinados: Sobre madera, mpio. de Xalapa, Rancho Guadalupe, col. G. Bills. 6-XII-01, CB767.

Distribución conocida: Jamaica (Crane y Dumont, 1975) y Cuba (MenaPortales y Mercado-Sierra, 1987; Holubová-Jechová y Mercado-Sierra, 1989). Crece sobre madera. 
Paraceratocladium silvestre R.F. Castañeda, 1987, Fungi Cubense II: 9.

Setas erectas, rectas, lisas, septadas, de color café oscuro, ápice agudo, 135-170 x 4.5-7.5 $\mu \mathrm{m}$. Conidióforos septados, adheridos a lo largo de la seta, de color café pálido, lisos, inconspicuos. Células conidiógenas monofialídicas, lageniformes, ampuliformes, con un collarín en el ápice, 8-13 x 3-6 $\mu \mathrm{m}$. Conidios aciculares, fusiformes, ligeramente curvados, con un septo transversal, lisos, hialinos, $15-20 \times 1-1.5 \mu \mathrm{m}$. Fig. 21.

Material examinado: Sobre hojarasca, mpio. de Xicochimalco, Cascadas de Texolo, cols. R. M. Arias y M. Reyes, 18-VI-01, CB769-1, CB769-2.

Distribución conocida: Cuba (Castañeda, 1987; Mercado-Sierra et al., 1997a). Crece sobre hojas y tallos muertos.

Phialocephala ivoriensis Zucconi et Onofri, 1984, Mycotaxon 20: 185-195.

Conidióforos solitarios, erectos, rectos o flexuosos, septados, lisos, de color café oscuro, hasta de $697 \times 17-26 \mu \mathrm{m}$, parte superior ramificada con 3 series de métulas coronadas por fiálides. Células conidiógenas terminales, fialídicas, en penicilios, determinadas, enteroblásticas, de color café claro, 12-15.5 × 2-3 $\mu \mathrm{m}$. Conidios ovalados, lisos, aseptados, en cadenas, embebidos en un mucílago, 3.5$4.5 \times 1.5-2 \mu \mathrm{m}$. Figs. $15 \mathrm{a}, \mathrm{b}$.

Materiales examinados: Sobre un rizoma de helecho, mpio. Cosautlán, Cosautlán, col. J. Mena-Portales, 6-VII-95, CB478-2. 1984).

Distribución conocida: Costa de Marfil, sobre hojarasca (Onofri y Zucconi,

Physalidium matsushimae R.F. Castañeda et W.B. Kendr., 1991, University of Waterloo Biology Series 35: 84.

Conidióforos erectos, rectos o flexuosos, septados, con ramas laterales que salen justo abajo de los septos, de color café oscuro en la base, aclarándose hacia el ápice, hasta $310 \mu \mathrm{m}$ de longitud y 5-7.5 $\mu \mathrm{m}$ de ancho en la base. Células conidiógenas distribuidas de la parte media del conidióforo hacia el ápice, monoblásticas discretas, subuladas, opuestas o verticiladas, de color café pálido, 7-10 x 1-1.5 $\mu \mathrm{m}$. Conidios formados por 3 células, la intermedia es fusiforme, ventricosa o bicónica, lisa, mamilada en la base, café obscura, 11-15 x 7-10 $\mu \mathrm{m}$, las dos células laterales son obturbinadas, lisas, subhialinas, 3.5-5 × 3.5-4.5 $\mu \mathrm{m}$. Fig. 18. 
Materiales examinados: Sobre hojas muertas, mpio. de Huatusco, Las Cañadas, cols. G. Heredia y R. Castañeda, 26-VII-99, CB770.

Distribución conocida: Cuba, sobre hojas de Cupania americana (Castañeda y Kendrick, 1991).

Pseudospiropes nodosus (Wallr.) M.B. Ellis, 1971, Dematiaceous Hyphomycetes, pág. 258.

$\equiv$ Helminthosporium nodosum Wallr., 1833, Fl. Crypt. Ger. 2: 165.

Conidióforos erectos, rectos o ligeramente flexuosos, simples, lisos, septados, paredes gruesas, de color café oscuro, 120-208 x 9-11 $\mu \mathrm{m}$. Células conidiógenas poliblásticas, terminales, cilíndricas, prominentemente cicatrizadas. Conidios fusiformes, naviculares, de color café oscuro, pseudoseptos 4 a 8 , base truncada y obscura, 34-51 x 15-17 $\mu \mathrm{m}$ en la parte más ancha y 5-6 $\mu \mathrm{m}$ en la base del conidio. Fig. 16.

Materiales examinados: Sobre bejuco, mpio. Calcahualco, Calcahualco, col. G. Rosas, 16-VI-95, CB475.

Distribución conocida: Canadá (Sutton, 1973), Nueva Zelanda (Hughes, 1978) y Malawi (Sutton, 1993). Prospera sobre sustratos leñosos.

Pyriculariopsis pleuroconidiophora Davydk. et Melnik, 1989, Mikologia i Fitopatologia 23: 112.

$\equiv$ Cacumisporium curvularioides R.F. Castañeda et W.B. Kendr., 1991, University of Waterloo Biological Series 35: 16.

Conidióforos simples, erectos, rectos, lisos, de color café oscuro en la base, más claros hacia el ápice, 392-527 × 7-14 $\mu \mathrm{m}$. Células conidiógenas terminales, integradas, simpodiales, con dentículos truncados. Conidios obturbinados, asimétricos, lisos, con 3 septos, las células de los extremos subhialinas a cafés claras, las células centrales más oscuras, $22-27 \times 11-12 \mu \mathrm{m}$. Fig. 5.

Materiales examinados: Sobre troncos en descomposición, mpio. Xalapa, Cerro Macuiltepetl, cols. G. Heredia, R. M. Arias y M. Reyes, 29-VIII-01, CB7541, CB754-2.

Distribución conocida: Krasnodar, en la antigua Rusia (Davydkina y Melnik, 1989) y Cuba (Castañeda y Kendrick, 1991). Especie lignícola. 
Sadasivania girisa Subram., 1957, J. Indian Bot. Soc. 36: 64-67.

Conidióforos simples, erectos, rectos o flexuosos, aseptados, lisos, de color café oscuro, hasta de $450 \mu \mathrm{m}$ de alto $\times 21-40 \mu \mathrm{m}$ en la base y 12-15 $\mu \mathrm{m}$ arriba de la base. Células conidiógenas acropleurógenas, situadas en la parte superior del conidióforo, poliblásticas, discretas, determinadas, subesféricas, lisas 0 verrucosas, con la parte distal fértil hialina y la parte basal estéril oscura, catenuladas, formando cabezuelas negras de 200-530 $\mu \mathrm{m}$ de diámetro. Conidios simples, esféricos, finamente equinulados, de color café oscuro rojizo, 7-9 $\mu \mathrm{m}$ de diámetro. Fig. 22.

Materiales examinados: Sobre tallos y hojas de maíz, mpio. Ixhuacán de Los Reyes, Ixhuacán de Los Reyes, col. A. Mercado-Sierra, 5-III-96, CB540-1.

Distribución conocida: La India, sobre hojas de pastos (Subramanian, 1957).

Spadicoides atra (Corda) S. Hughes, 1958, Can. J. Bot. 36: 805.

Conidióforos erectos, rectos o flexuosos, septados, lisos, ápice redondeado, de color café oscuro, aclarándose hacia la parte superior, $152 \times 2-3 \mu \mathrm{m}$. Células conidiógenas terminales e intercaladas, integradas, tréticas. Conidios obovoides 0 claviformes, con los extremos redondeados, algunos con la base truncada, lisos, aseptados, de color café oscuro, 4.5-5.5 x 2.5-3 $\mu \mathrm{m}$. Fig. 23.

Materiales examinados: Sobre tronco, mpio. Calcahualco, Calcahualco, col. J. Mena-Portales, 16-VI-95, CB439-1.

Distribución conocida: Estados Unidos (Ellis, 1963), Canadá (Sutton, 1973), Japón (Matsushima, 1975), Nueva Zelanda (Hughes, 1978), Hungría, la antigua Checoslovaquia (Holubová-Jechová, 1979, 1982b), India (Rao y de Hoog, 1986), Malawi (Sutton, 1993) y Cuba (Mercado-Sierra, et al., 1997b). Común sobre madera y ramas muertas.

Spadicoides obovata (Cooke et Ellis) S. Hughes, 1958, Can. J. Bot. 38: 806. EAcrothecium obovatum Cooke et Ellis, 1876, Grevillea 5: 50, 1876.

ESpondylocladium obovatum (Cooke et Ellis) S. Hughes, 1953, Can. J. Bot., 31: 634 .

Conidióforos erectos, rectos o flexuosos, septados, lisos, con numerosos poros a lo largo y en la parte apical, de color café oscuro, 85-205 x 3.5-5 $\mu \mathrm{m}$. Células conidiógenas terminales e intercaladas, integradas, tréticas. Conidios obovoides 0 
claviformes, ampliamente redondeados en el ápice, ligeramente cónico-truncados en la base, lisos, de color café oscuro, con dos gruesos septos, de color café oscuro $12-14 \times 7-7.5 \mu \mathrm{m}$. Fig. 25a, b.

Materiales examinados: Sobre ramas muertas, mpio. Xalapa, Rancho Guadalupe, cols. G. Heredia, R. M. Arias y M. Reyes, 29-XI-01, CB771.

Distribución conocida: Puerto Rico (Ashford y Ciferri, 1930), Canadá, Estados Unidos (Ellis, 1963), Japón (Matsushima, 1975), Nueva Zelanda (Hughes, 1978), Cuba (Mercado-Sierra, 1981) y Polonia (Holubová-Jechová, 1982b). Crece sobre ramas y madera muerta.

Sporidesmium altum (Preuss) M.B. Ellis, 1958, Mycological Papers 70: 46-48.

$\equiv$ Helminthosporium altum (Preuss) Sacc., 1886, Sylloge Fungorum 4: 425.

$\equiv$ Podoconis alta (Preuss) Mason et S. Hughes, 1953, The Naturalist, London, No. 846, July-September 1953, p. 119.

$\equiv$ Helminthosporium turbinatum Berkeley et Broome, 1851, Ann. Mag. Nat. Hist. ser. 2, 7: 98.

Conidióforos, erectos, rectos o ligeramente flexuosos, solitarios, septados, lisos, de color café oscuro, más pálidos hacia el ápice, 79-139 x 4-5 $\mu \mathrm{m}$. Células conidiógenas terminales, integradas, percurrentes. Conidios cónicos u obpiriformes, rectos o curvos, truncados en la base, septados, lisos, con 5-6 septos, célula basal más grande que las otras células, de color café oscuro con el extremo más claro, 33-42 x 14-18 $\mu \mathrm{m}$. Fig. 26a, b.

Materiales examinados: Sobre ramas muertas, mpio. Ixhuacán de Los Reyes, Ixhuacán de Los Reyes, col. A. Mercado-Sierra, 5-III-96, CB539-1.

Distribución conocida: Inglaterra (Ellis, 1958), Japón (Matsushima, 1975) y la India (Rao y de Hoog, 1986). Sobre madera y ramas muertas.

Sporoschisma saccardoi anamorfo de Melanochaeta hemipsila (Berk. et Br.) Müll, Harr et Sulmont, 1969, Revue de Mycologie 33: 377.

Colonias dispersas, de color café oscuro a negruzcas, superficiales, velutinas, compuestas de conidióforos intercalados con hifas capitadas. Conidióforos erectos, rectos, lisos, pared gruesa, negruzcos, hasta de $255 \mu \mathrm{m}$ de alto por 15-19 $\mu \mathrm{m}$ en la parte más ancha. Hifas capitadas subuladas, erectas, rectas o flexuosas, lisas, septadas, ápice inflado, de color café oscuro en la base y aclarándose hacia el ápice, 175-251 x 8-10 $\mu \mathrm{m}$ en la parte apical. Conidios cilíndricos, con los extremos aplanados, lisos, con 5 septos, de color café oscuro, células distales más claras que las intermedias de paredes gruesas, 52-68 x 12-15 $\mu \mathrm{m}$. Ascoma superficial, 
piriforme, hasta de $560 \mu \mathrm{m}$ de alto y $460 \mu \mathrm{m}$ de ancho, pared lisa. Ascas unitunicadas, cilíndricas o claviformes, con ocho ascosporas, ápice con anillo refractario inamiloide. Ascosporas fusiformes, con 5 septos transversales, curvadas, células centrales más oscuras que las de los extremos, 30-40 x 7.5-10 $\mu \mathrm{m}$. Figs. 28a-c.

Materiales examinados: Sobre troncos caídos, mpio. Coatepec, Rancho Viejo, cols. G. Heredia y J. Mena-Portales, 18-V-02, CB772-1, CB772-1.

Distribución conocida: Italia, Canadá (Nag Raj y Kendrick, 1975), Taiwan (Matsushima, 1980), Australia (Matsushima, 1989; Goh et al., 1997), Perú (Matsushima, 1993), Hong Kong, Malasia y Sudáfrica (Goh et al., 1997), Cuba (Minter et al., 2001) y Tailandia (Sivichai et al., 2000). Prospera en sustratos lignícolas sumergidos. El teleomorfo, Melanochaeta hemipsila ha sido identificado en materiales de Australia, Guyana Francesa, Sri Lanka y Tailandia (Sivichai et al., 2000).

Subulispora longirostrata Nawawi et Kutubuth., 1987, Mycotaxon 30: 459-463.

Conidióforos erectos, rectos o flexuosos, septados, lisos, de color café claro, $45-52 \times 3.5-4 \mu \mathrm{m}$. Células conidiógenas integradas, determinadas, simpodiales, situadas en la parte terminal del conidióforo. Conidios cónicos, con la base truncada y la parte apical aguda, lisos, con 3 septos, hialinos, $23-26.5 \times 2-2.5 \mu \mathrm{m}$, con un apéndice en la parte apical, 13-17 $\mu \mathrm{m}$ de largo. Fig. 30.

Materiales examinados: Sobre hoja sumergida, mpio. Xalapa, Rancho Guadalupe, cols. G. Heredia, R. M. Arias y M. Reyes, 29-XI-01, CB773.

Distribución conocida: Malasia (Nawawi y Kutubutheen, 1987) y Brasil (Gusmao, 2001). Sobre hojas muertas.

\section{AGRADECIMIENTOS}

El presente trabajo se realizó gracias al apoyo financiero de la Comisión Nacional para el Conocimiento y Uso de la Biodiversidad (Proyectos: P030 y B139) y del Instituto de Ecología, A.C. (INECOL p02-07). También se agradece el auxilio técnico de la Biól. Greta Rosas Saito.

\section{LITERATURA CITADA}

Ashford, B. K. y R. Ciferri. 1930. A new variety of Acrothecium obovatum. Mycologia 22:180185. 
Barron, G. L. 1977. The genera of Hyphomycetes from soil. Ed. Krieger. Nueva York. 364 pp.

Bhat, D. J. y B. C. Sutton. 1985. Some phialidic Hyphomycetes from Ethiopia. Trans. Br. Mycol. Soc. 84(4): 723-730.

Cabello, M., C. Cazau y A. Arambarri. 1993. Estudio de los Hyphomycetes del Río Santiago. VI. (Buenos Aires, Argentina). Bol. Soc. Argent. Bot. 29(1-2): 11-14.

Castañeda, R. F. 1985. Deuteromycotina de Cuba. Hyphomycetes II. Instituto de Investigaciones Fundamentales en Agricultura Tropical "Alejandro de Humboldt". La Habana, Cuba. 23 pp.

Castañeda, R. F. 1987. Fungi Cubense II. Instituto de Investigaciones Fundamentales en Agricultura Tropical "Alejandro de Humboldt". La Habana, Cuba. 22 pp.

Castañeda, R. F. y G. R. W. Arnold. 1985. Algunos hongos nuevos para Cuba. Revista del Jardín Botánico Nacional, Universidad de La Habana 6: 55-56.

Castañeda, R. F. y B. Kendrick. 1991. Ninety-nine conidial fungi from Cuba and three from Canada. University of Waterloo Biology Series 35: 1-132.

Chacón, S. y R. Medel. 1993. Los hongos (principalmente macromicetos) registrados en el bosque mesófilo de montaña de México. Universidad Autónoma de Nuevo León. Fac. de Ciencias Forestales. Reporte Científico No. Especial 13: 61-110.

Crane J. L. y K. P. Dumont. 1975. Hyphomycetes from the West Indies and Venezuela. Can. J. Bot. 53(9): 845-851.

Crane J. L. y D. L. Schocknecht. 1982. Hyphomycetes from fresh water swamps and hammocks. Can. J. Bot. 60: 369-378.

Czeczuga, B. y M. Orlowska. 1997. Hyphomycetes in the water of peat-moss (Sphagnum). Roczniki Akademii Medycznej w Bialymstoku 42: 183-190.

Davydkina, T. A. y V. A. Melnik. 1989. Two new Hyphomycetes from the genera Cordana and Pyriculariopsis. Mikologia i Fitopatología 23: 110-113.

Dickinson, C. H. 1968. Gliomastix Guéguen. Mycol. Pap. 115: 15-16.

Ellis, M. B. 1958. Clasterosporium and some allied Dematiaceae-Phragmosporae. I. Mycol. Pap. 70: 1-90.

Ellis, M. B. 1963. Dematiaceous Hyphomycetes. V. Mycol. Pap. 93: 1-33.

Ellis, M. B. 1971. Dematiaceous Hyphomycetes. Commonwealth Mycological Institute. Kew. $608 \mathrm{pp}$.

Ellis, M. B. 1976. More Dematiaceous Hyphomycetes. Commonwealth Mycological Institute. Kew. 507 pp.

Goh, T. K., W. H. Ho., K. D. Hyde y T. Umali. 1997. New records and species of Sporoschisma and Sporoschismopsis from submerged wood in the tropics. Mycol. Res. 101(11): 1295-1307.

Gusmao, L. F. P. 2001. Hyphomycetes from leaf litter of Miconia cabussus in the Brazilian Atlantic rain forest. Mycotaxon 79: 201-213.

Hawksworth, D. L., P. M. Kirk, B. C. Sutton y D. N. Pegler. 1995. Ainsworth \& Bisby's dictionary of the fungi. 8a. ed. International Mycological Institute. Wallingford. $616 \mathrm{pp}$.

Hennebert, G. L. y L. K. Weresub. 1979. Terms for states and forms of fungi, their names and types. In: Kendrick B. (ed.). The whole fungus. Vol. 1. National Museum of Natural Sciences. Calgary. pp. 27-41.

Holubová-Jechová, V. 1979. Lignicolous and some other saprophytic Hyphomycetes from Hungary. Ceská Mykol. 33: 137-149.

Holubová-Jechová, V. 1982a. New or interesting phialidic Hyphomycetes from Cuba. Mycotaxon 15: 277-292. 
Holubová-Jechová, V. 1982b. Lignicolous Hyphomycetes from Czechoslovakia. 6. Spadicoides and Diplococcium. Folia Geobot. Phytotax. Praha 17: 295-327.

Holubová-Jechová, V. 1987. Studies on Hyphomycetes from Cuba V. Six new species of Dematiaceous Hyphomycetes from Havana province. Ceská Mykol. 41(1): 29-36.

Holubová-Jechová, V. y A. Mercado-Sierra. 1989. Hyphomycetes from Loma de la Coca and some localities of La Habana and Matanzas province, Cuba. Acta Bot. Cub. 76: 1-15.

Hughes, S. J. 1951. Studies on micro-fungi XIII. Beltrania, Ceratocladium, Diplorhinotrichum, and Hansfordiella (gen. nov.). Mycol. Pap. 47: 1-25.

Hughes, S. J. 1953. Fungi from the Gold Coast. II. Mycol. Pap. 50: 65-66.

Hughes, S. J. 1978. New Zealand fungi. 25. Miscellaneous species. New Zealand J. of Bot. 16: $311-370$.

Hughes, S. J. y B. Kendrick. 1968. New Zealand fungi. 12. Menispora, Codinaea, Menisporopsis. New Zealand J. of Bot. 6 : 323-375.

Kirk, P. M. 1986. New or interesting microfungi XV. Miscellaneous Hyphomycetes from the British Isles. Trans. Br. Mycol. Soc. 86(3): 409-422.

Kirk, P. M. y B. C. Sutton. 1985. A reassessment of the anamorph genus Chaetopsina (Hyphomycetes). Trans. Br. Mycol. Soc. 85(4): 709-718.

Kuthubutheen, A. J. y A. Nawawi. 1994. Henicospora longissima sp. nov., Obeliospora triappendiculata sp. nov., Paraulocladium fabisporum sp. nov. and other Hyphomycetes from Malaysia. Mycol. Res. 98(6): 677-685.

Matsushima, T. 1971. Microfungi of the Solomon Islands and Papua-New Guinea. Published by the author. Kobe. $78 \mathrm{pp}$.

Matsushima, T. 1975. Icones microfungorum a Matsushima lectorum. Published by the author. Kobe. 209 pp.

Matsushima, T. 1980. Mycological Memories No. 1. Published by the author. Kobe. 82 pp.

Matsushima, T. 1981. Matsushima Mycological Memories No. 2. Published by the author. Kobe. 68 pp.

Matsushima, T. 1987. Matsushima Mycological Memories No. 5. Published by the author. Kobe. 100 pp.

Matsushima, T. 1989. Matsushima Mycological Memories No. 6. Published by the author. Kobe. 100 pp.

Matsushima, T. 1993. Matsushima Mycological Memories No. 7. Published by the author. Kobe. 75 pp.

McKenzie, E. H. C. 1995. Dematiaceous Hyphomycetes on Pandanaceae. 5. Sporidesmium sensu lato. Mycotaxon 56: 9-29.

Melnik, V. A. 1998. Some notes on Deuteromycetes from tropical countries. Mikologia i Fitopatologia 32: 32-43.

Melnik, V. A. 2000. Definitorium fungorum Rossiae. Classis Hyphomycetes. Fasc. 1. Fam. Dematiaceae. Nauka. San Petersburgo. 370 pp.

Mena-Portales, J. y A. Mercado-Sierra. 1987. Algunos hifomicetes de la provincia Ciudad de La Habana, Cuba. Ecología y Sistemática. Academia de Ciencias de Cuba 17: 1-17.

Mercado-Sierra, A. 1981. Lista preliminar de hifomicetes dematiáceos de la estación Ecológica de Sierra del Rosario y zonas adyacentes. Acta Bot. Cubana 6: 1-6.

Mercado-Sierra, A. y J. Mena-Portales. 1986. Hifomicetes de topes de collantes Cuba I. (Especies holoblásticas). Acta Bot. Hung. 32(1-4): 189-205. 
Mercado-Sierra, A., Holubová-Jechová y J. Mena-Portales. 1997a. Hifomicetes dematiáceos de Cuba. Enteroblásticos. Monografie XXIII. Museo Regionale di Scienze Naturali Torino. Turín 388 pp.

Mercado-Sierra, A., J. Gené y J. Guarro. 1997b. Some Costa Rican Hyphomycetes. I. Nova Hedwigia 64: 111-121.

Mercado-Sierra, A., J. Gené, M. J. Figueras, K. Rodríguez y J. Guarro. 1998. New or rare Hyphomycetes from Cuba. IX. Some species from Pinar del Rio Province. Mycotaxon 67: 417-426.

Minter, D. W., M. Rodríguez-Hernández y J. Mena-Portales (eds.). 2001. Fungi of the Caribbean. FMDS Publisher. Londres. 946 pp.

Morgan-Jones, G. y J. M. McKemy. 1992. Studies in the genus Cladosporium sensu lato. VI. Concerning Cladosporium vignae, causal organism of leaf of pod spot of Cowpea (Vigna unguiculata) of leaf blight of Lespedeza bicolor. Mycotaxon 43: 19-20.

Morris, E. F. 1978. Belizean Hyphomycetes. Mycotaxon 7(2): 265-274.

Nag Raj, T. R. y B. Kendrick. 1975. A monograph of Chalara and allied genera. Department of Biology. University of Waterloo. Waterloo, Ontario. $200 \mathrm{pp}$.

Nawawi, A. y A. J. Kuthubutheen. 1987. A new species of the genus Subulispora. Mycotaxon 30: $459-463$.

Onofri, S. y L. Zucconi. 1984. Two new species of the genus Phialocephala. Mycotaxon 20(1): 185-195.

Rao, V. y K. I. Varghese. 1981. Some new or interesting Hyphomycetes from India. Proc. Nat. Acad. Sci. India 51(B) III: 240-244.

Rao, V. y G. S. de Hoog. 1986. New or critical Hyphomycetes from India. Studies in Mycol. 28: 1-83.

Siviachi, S., N. L. Hywel-Jones y S. Somrithipol. 2000. Lignicolous freshwater Ascomycota from Thailand: Melanochaeta and Sporchisma anamorphs. Mycol. Res. 104(4): 478485.

Subramanian, C. V. 1957. Hyphomycetes-III. Two new genera, Dwayaloma and Sadasivania. J. Indian Bot. Soc. 36(1): 61-67.

Subramanian, C. V. 1958. Hyphomycetes-VI. Two new genera, Edmundmasonia and lyengarina. J. Indian Bot. Soc. 37(3): 401-407.

Subramanian, C. V. 1992. A reassessment of Sporidesmium (Hyphomycetes) and some related taxa. Proc. Indian Natn. Sci. Acad. B 58(4): 179-190.

Subramanian, C. V. y D. J. Bhat. 1987. Hyphomycetes from South India I. Some new taxa. Kavaka 15(1,2): 41-74.

Sutton, B. C. 1973. Hyphomycetes from Manitoba and Saskatchewan, Canada. Mycol. Pap. 132: 1-143.

Sutton, B. C. 1976. Species of Hemibeltrania Piroz. and Dischloridium gen. nov. Kavaka 4: 43-50.

Sutton, B. C. 1993. Mitosporic fungi from Malawi. Mycol. Pap. 167: 1-93.

Sutton, B. C. y C. S. Hodges. 1976. Eucalyptus microfungi: Some setose Hyphomycetes with phialides. Nova Hedwigia 27: 343-352.

Thian-Yu, Z. y B. Kendrick. 1990. A reassessment of Botryosporium (Hyphomycetes). Acta Mycol. Sin. 9(1): 31-40. 
Heredia et al.: Hongos conidiales del bosque mesófilo de montaña de Veracruz
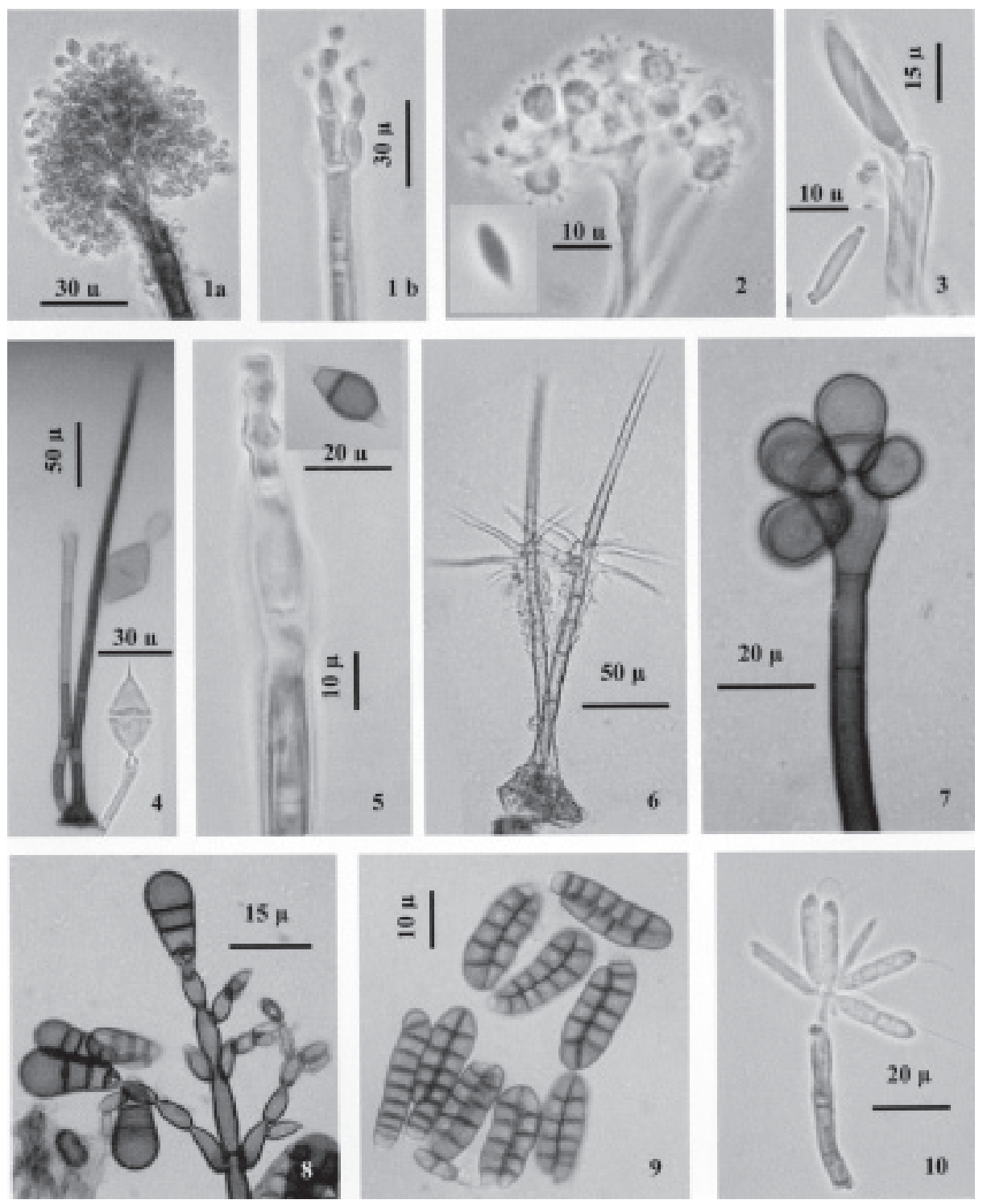

Figs. 1-10. 1. Alysidiopsis pipsissewae: a. zona apical del conidióforo con esporas. b. células conidiógenas con conidios esféricos. 2. Botryosporium longibrachiatum: zona apical del conidióforo con células conidiógenas y conidio. 3. Cladosporium vignae: célula conidiógena con cicatrices y conidios. 4. Beltrania malaiensis: seta simple, conidióforo y conidio con banda trasversal hialina. 5. Pyriculariopsis pleuroconidiophora: conidióforo con célula conidiógena simpodial y conidio obturbinado con tres septos. 6. Chaetopsina splendida: seta con ramas divergentes. 7. Cordana musae: zona apical del conidióforo con conidios piriformes. 8. Edmundmasonia pulchra: zona apical del conidióforo con células conidiógenas fiálidicas y conidios adheridos. 9. Eversia parvula: conidios muriformes. 10. Hyphodiscosia jaipurensis: conidióforo con conidios. 
Acta Botanica Mexicana (2004), 66: 1-22
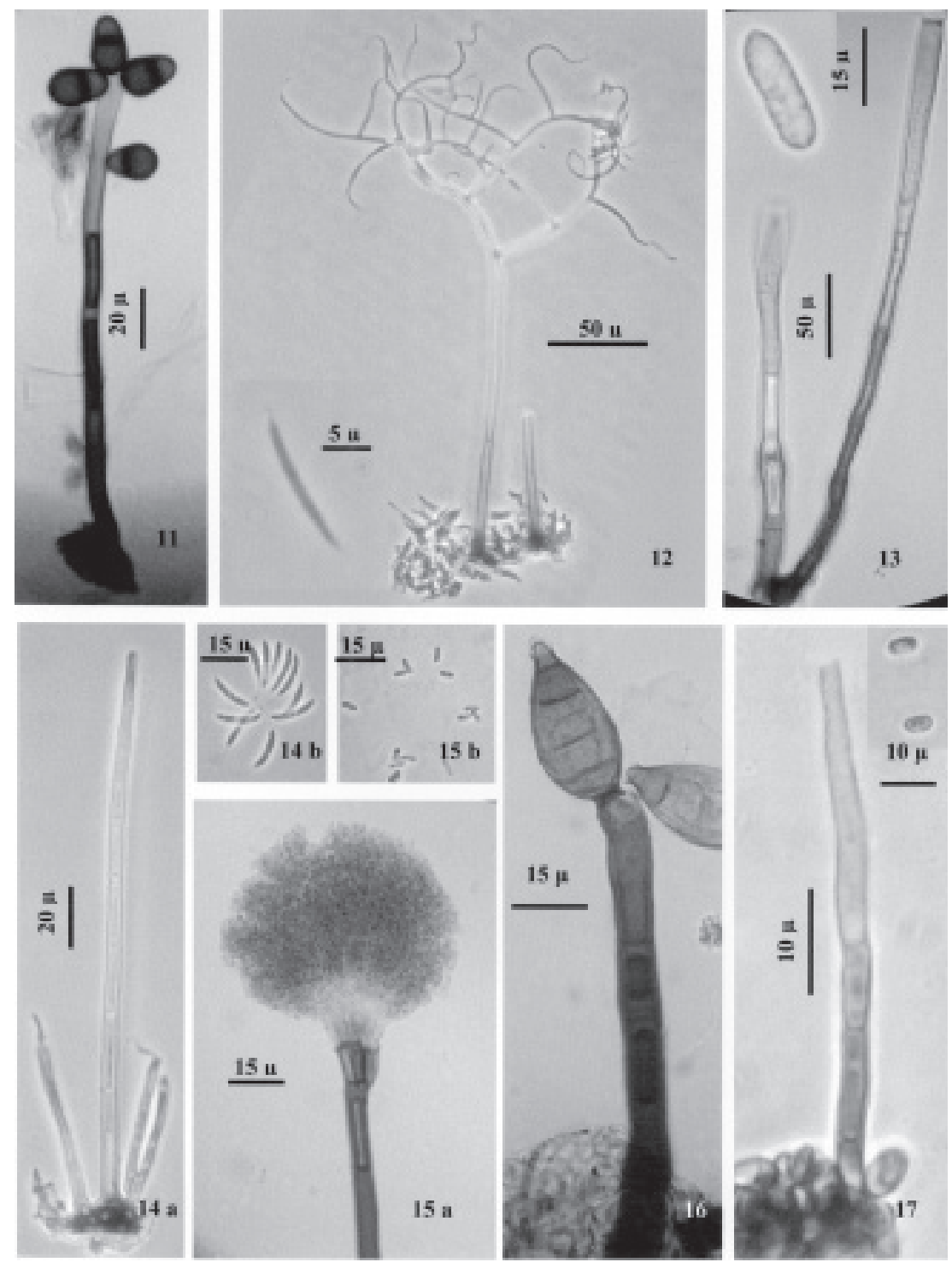

Figs. 11-17. 11. Cordana crassa: conidióforo con conidios obpiriformes. 12. Gyrothrix podosperma var. falcatispora: seta ramificada con la base fértil y conidio falciforme. 13. Dischloridium laeense: conidióforo y conidio cilíndrico. 14. Dictyochaeta anamorfo de Chaetosphaeria dingleyae: a. seta y conidióforos. b. conidios fusiformes. 15. Phialocephala ivoriensis: a. zona apical del conidióforo con métulas y conidios embebidos en un mucílago. b. conidios ovalados. 16. Pseudospiropes nodosus: conidióforo con conidio adherido. 17. Acremonium anamorfo de Wallrothiella subiculosa: conidióforo y conidios elipsoidales. 
Heredia et al.: Hongos conidiales del bosque mesófilo de montaña de Veracruz
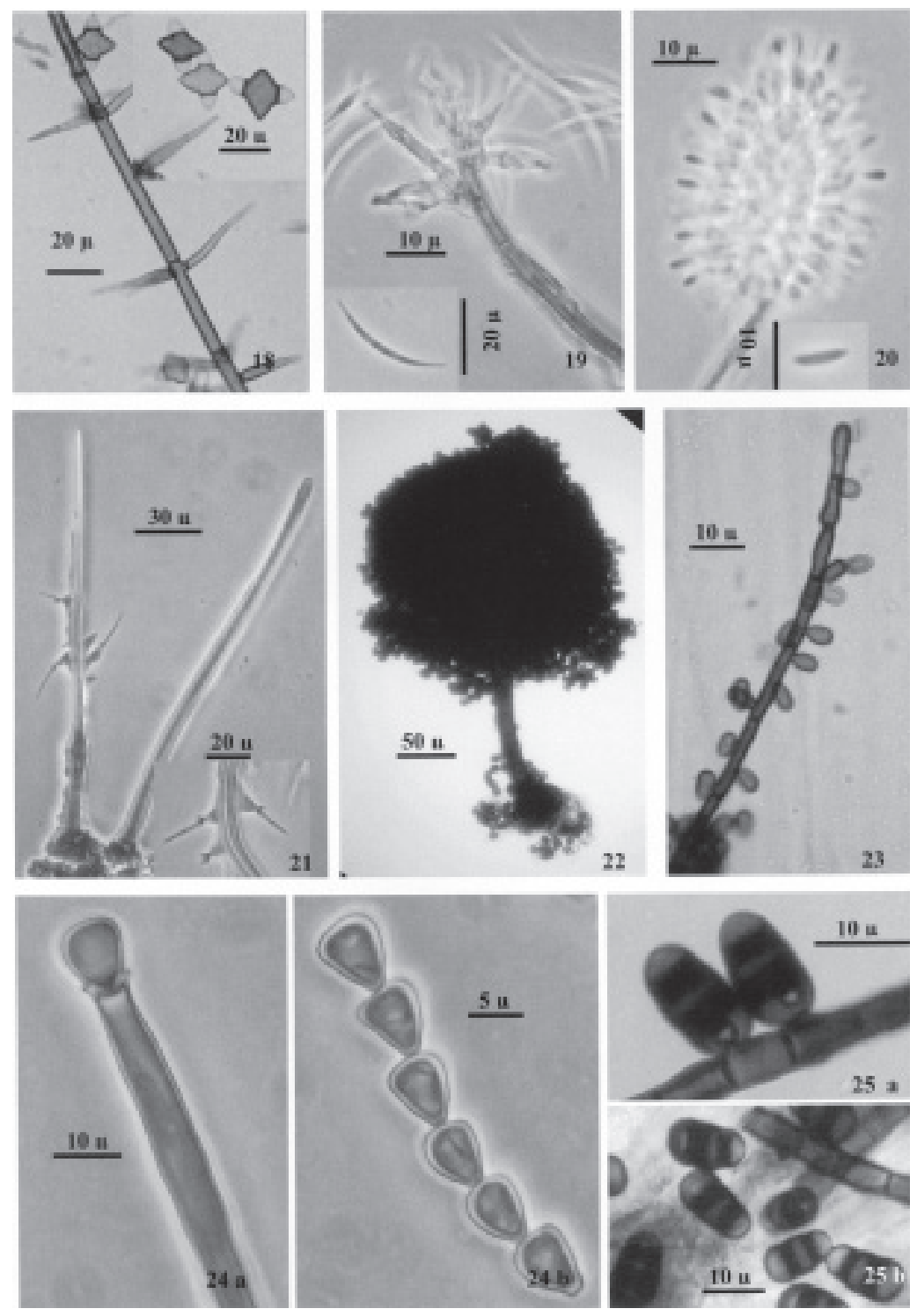

Figs. 18-25. 18. Physalidium matsushimae: zona apical del conidóforo con células conidiógenas subuladas verticiladas y conidios. 19. Idriella rara: zona apical del conidióforo con células conidiógenas y conidios falciformes. 20. Masoniomyces claviformis: células conidiógenas cubriendo la parte claviforme del conidióforo con conidios fusiformes. 21. Paraceratocladium silvestre: setas simples con conidióforos, conidios fusiformes. 22. Sadasivania girisa: conidióforo con conidios formando cabezuelas negras. 23. Spadicoides atra: conidióforo con conidios obovoides adheridos. 24. Catenularia kalakadensis: a. conidióforo con conidio en formación. b. conidios cuneiformes en cadena. 25. Spadicoides obovata: a. conidióforo con conidios obovoides adheridos. b. conidios con septos negros. 

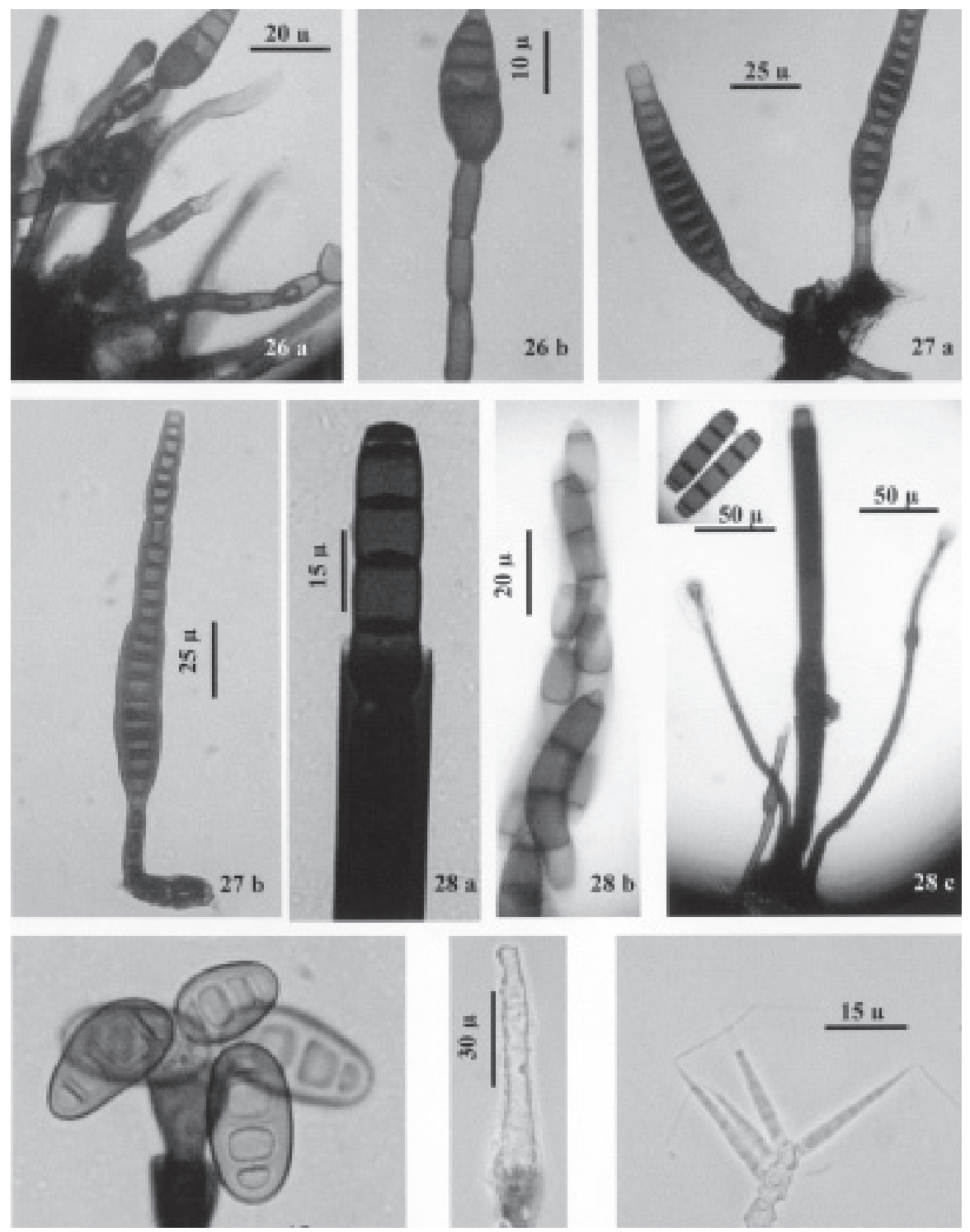

Figs. 26-30. 26. Sporidesmium altum: a. conidióforos. b. conidio obpiriforme adherido al conidióforo. 27. Ellisembia adscendens: a. conidios jóvenes. b. conidióforo con conidio maduro. 28. Sporoschisma anamorfo de Melanochaeta hemipsila: a. conidio saliendo del conidióforo. b. asca con ascosporas. c. conidióforo con hifas capitadas y conidios. 29. Exserticlava vasiformis: a. célula conidiógena y conidios elipsoides. b. conidióforo con extensión. 30. Subulispora longirostrata: conidióforo con células conidiógenas simpodiales y conidios cónicos con una sétula en la parte apical. 